\title{
8
}
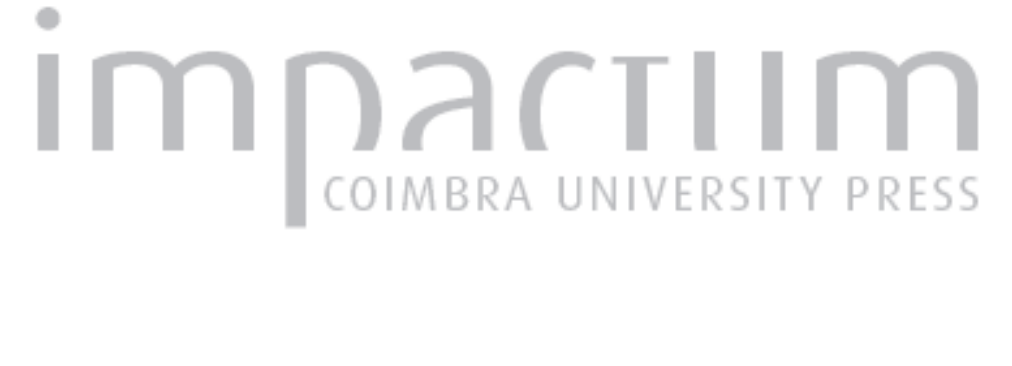

\section{Estudos literários digitais: projetos, métodos, ferramentas}

Autor(es): $\quad$ Ministro, Bruno

Publicado por: Centro de Literatura Portuguesa; Imprensa da Universidade de Coimbra

URL persistente:

URI:http://hdl.handle.net/10316.2/38733

DOI:

DOI:http://dx.doi.org/10.14195/2182-8830_4-1_10

Accessed : $\quad$ 26-Apr-2023 14:35:36

A navegação consulta e descarregamento dos títulos inseridos nas Bibliotecas Digitais UC Digitalis, UC Pombalina e UC Impactum, pressupõem a aceitação plena e sem reservas dos Termos e Condições de Uso destas Bibliotecas Digitais, disponíveis em https://digitalis.uc.pt/pt-pt/termos.

Conforme exposto nos referidos Termos e Condições de Uso, o descarregamento de títulos de acesso restrito requer uma licença válida de autorização devendo o utilizador aceder ao(s) documento(s) a partir de um endereço de IP da instituição detentora da supramencionada licença.

Ao utilizador é apenas permitido o descarregamento para uso pessoal, pelo que o emprego do(s) título(s) descarregado(s) para outro fim, designadamente comercial, carece de autorização do respetivo autor ou editor da obra.

Na medida em que todas as obras da UC Digitalis se encontram protegidas pelo Código do Direito de Autor e Direitos Conexos e demais legislação aplicável, toda a cópia, parcial ou total, deste documento, nos casos em que é legalmente admitida, deverá conter ou fazer-se acompanhar por este aviso.

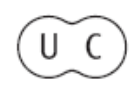




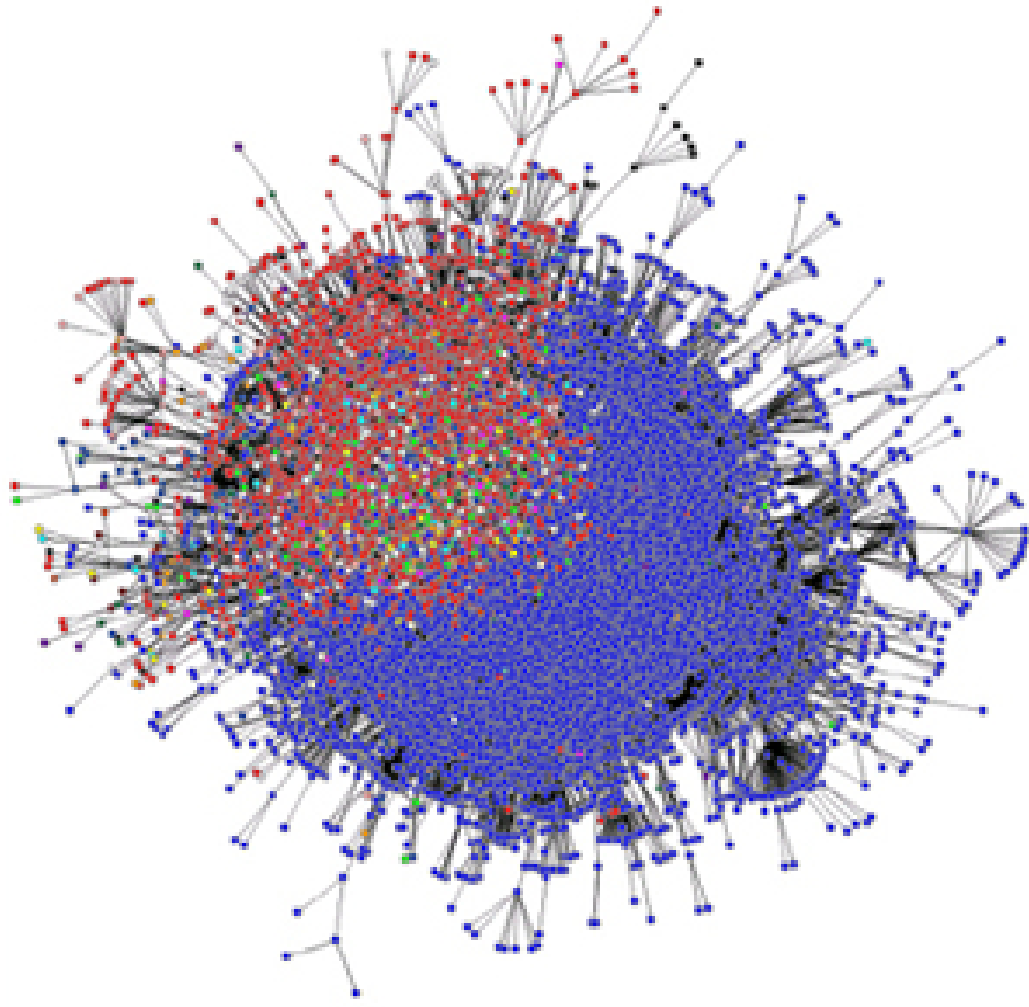

Vol. 4.1 (2016) ISSN 2182-8830

'Estudos Literários Digitais 1'

Manuel Portela e António Rito Silva (orgs.) 


\title{
Estudos Literários Digitais: \\ Projetos, Métodos, Ferramentas \\ BRUNO MINISTRO
}

\author{
CLP | Universidade de Coimbra
}

Bolseiro FCT

ELMCIP Anthology of European Electronic Literature
http://anthology.elmcip.net/
Editores: Maria Engberg, Talan Memmott e David Prater.

Editores: Maria Engberg, Talan Memmott e David Prater. Data de publicação: 2012. Blekinge Institute of Technology. Acesso aberto Cobras da antologia distribuidas sob licença Creative Commons].

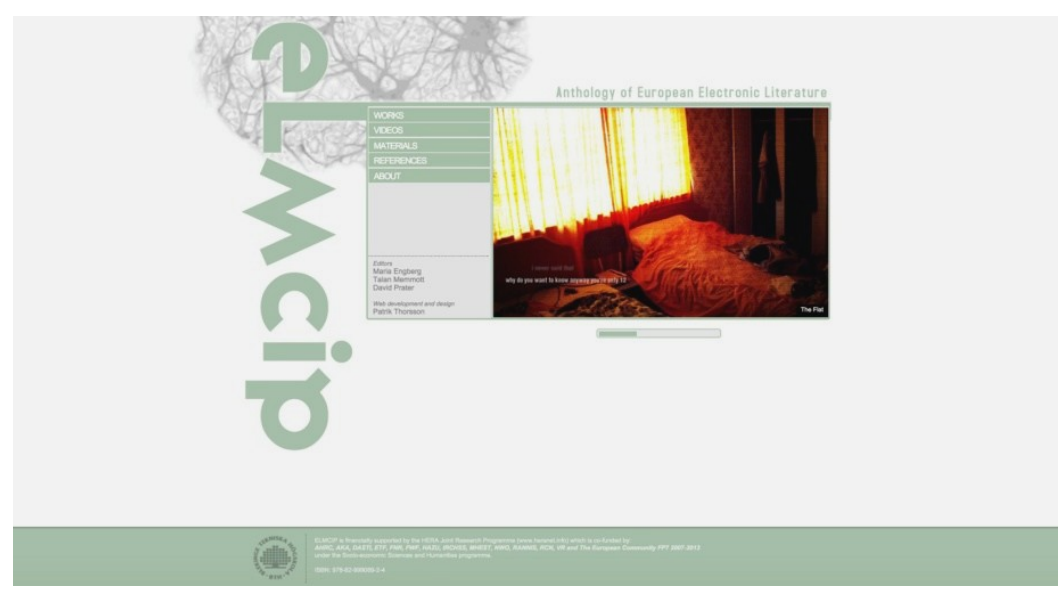

A ELMCIP Anthology of European Electronic Literature agrega obras de literatura eletrónica de artistas europeus e tem como finalidade fornecer a professores, estudantes e ao público em geral material pedagógico adequado ao reconhecimento de algumas das formas literárias digitais produzidas na Europa. A antologia foi publicada em 2012, como resultado do projeto de investigação Electronic Literature as a Model of Creativity and Innovation in Practice (ELMCIP), procurando oferecer uma visão diversificada do campo, plural no que diz respeito aos países representados, às línguas das obras e aos recursos e formas expressivas usados. Os princípios que regeram a seleção das 18 obras patentes na antologia e que, de certo modo, se assumem como os critérios da antologia, são os de criar um material representativo da diversidade cultural e formal, evidenciando a sua relevância histórica e pedagógica. A componente pedagógica tem particular destaque no portal da Anthology of 
European Electronic Literature, sendo-lhe dedicadas três secções específicas: "vídeos", "materiais" e "referências". No seu conjunto, estas secções fornecem um robusto corpo de leituras e materiais audiovisuais, com ligação à ELMCIP Knowledge Base, o que não se materializa apenas num valioso recurso pedagógico para a sala de aula, como constitui também um importante instrumento para o reconhecimento público das práticas que têm lugar fora dos centros históricos da literatura eletrónica.

Bibliografia relacionada:

Rettberg, Scott (2016). "Electronic Literature as Digital Humanities." A New Companion to Digital Humanities. Eds. Susan Schreibman, Ray Siemens \& John Unsworth. Chichester: Wiley-Blackwell. 127-136.

Rettberg, Scott, Patricia Tomaszek \& Sandy Baldwin, eds. (2015). Electronic Literature Communities. Morgantown, WV: Center for Literary Computing / ELMCIP.

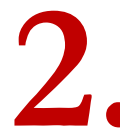

The Deena Larsen Collection

http://mith.umd.edu/larsen/

Diretor: Matthew Kirschenbaum. Data de publicação: 2007-2011. Maryland Institute for Technology in the Humanities. Acesso aberto [materiais do portal distribuídos sob licença Creative Commons].

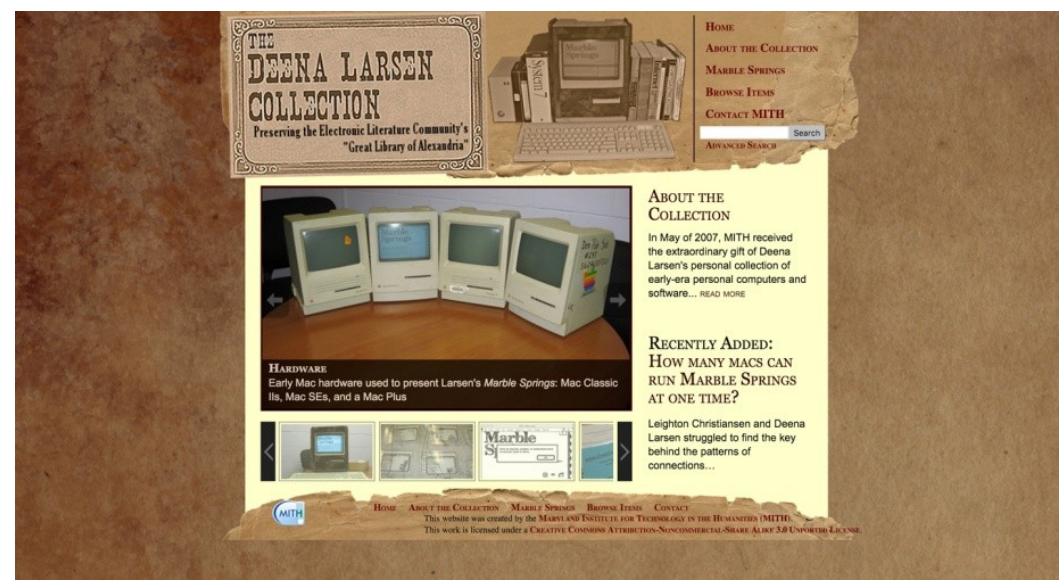

O projeto The Deena Larsen Collection teve início em maio de 2007, depois de Deena Larsen ter doado a sua coleção particular ao MITH. Como artista da primeira geração da computação pessoal, o seu arquivo constitui um importante legado para entender as primeiras experiências no domínio da literatura eletrónica e hipertextual. Movido pelo objetivo de curar e preservar este acervo histórico, The Deena Larsen Collection é composto por obras da autora, em várias versões, algumas delas inéditas, mas também por obras de artistas como William Dickey, Stephanie Strickland, M.D. Coverley, Mikael And, Jim 
Rosenberg, Michael Joyce, Stuart Moulthrop, George Landow, entre outros. Fazem ainda parte do espólio físico de The Deena Larsen Collection um computador Apple Mac Classic II e respetivos periféricos, bem como disquetes, CDs, ZIP drives, manuais de utilizador e, ainda, um amplo número de recortes de jornal, manuscritos e várias publicações relacionadas com o tema. $\mathrm{O}$ sítio web do projeto fornece representações digitais dos itens que figuram na coleção física, catalogados com identificadores únicos e aqui disponíveis para consulta prévia dos investigadores interessados no acervo. Uma das secções do portal é dedicada a Marble Springs (1993), a mais famosa obra da autora, contendo um breve texto de introdução, representações digitais de materiais dos processos de criação e disseminação da obra e uma versão em vídeo de demonstração da navegabilidade a partir da emulação de Marble Springs. É objetivo declarado dos responsáveis de The Deena Larsen Collection que o projeto permita desenvolver ferramentas e recursos que possam migrar para outras plataformas e sistemas, garantindo a sua integridade, tanto material como criativa, no sentindo de promover um melhor conhecimento das práticas e objetos históricos pioneiros no uso expressivo do computador.

Bibliografia relacionada:

Kirschenbaum, Matthew G. (2016). "Ancient Evenings: Retrocomputing in the Digital Humanities." A New Companion to Digital Humanities. Eds. Susan Schreibman, Ray Siemens \& John Unsworth. Chichester: WileyBlackwell. 185-197.

Emerson, Lori (2014). Reading Writing Interfaces: From the Digital to the Bookbound. Minneapolis: University of Minnesota Press. 


\section{Literary Studies in the Digital Age: An Evolving Anthology https://dlsanthology.commons.mla.org/ \\ Editores: Kenneth M. Price e Ray Siemens. Data de publicação: 2013-2016 [em curso]. Modern Language Association. Acesso aberto.}

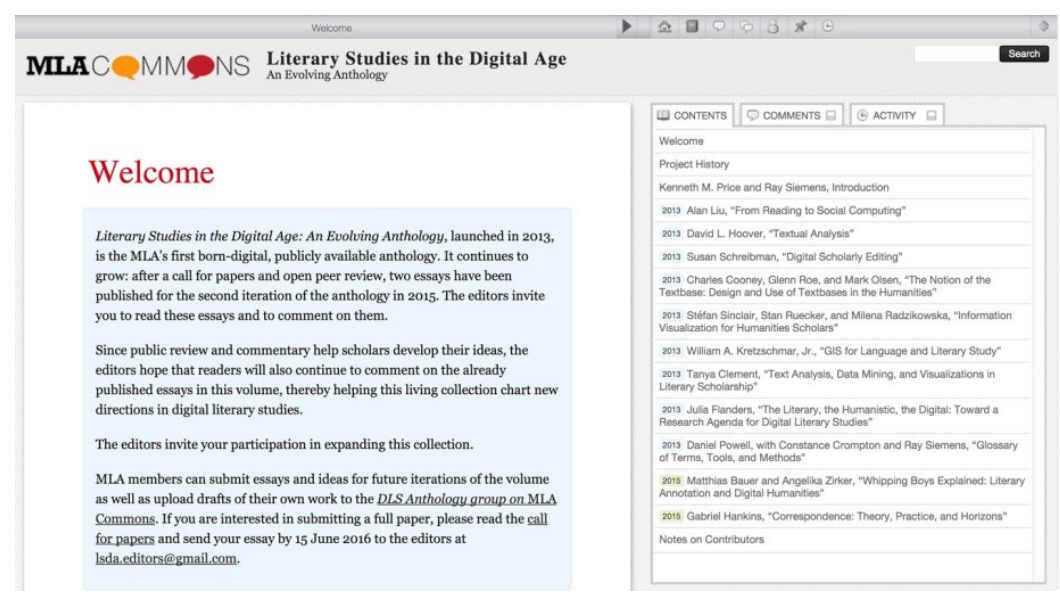

Literary Studies in the Digital Age assume-se, como o subtítulo indica, enquanto antologia em contínua evolução, interiorizando a lógica reticular e colaborativa da Web 2.0 como espaço de publicação. Debruçada sobre temas dos estudos literários na sua intersecção com os meios, técnicas e ferramentas digitais, é seu intuito construir, de forma paulatina e sustentada, uma antologia de textos de referência. O projeto foi lançado em 2013, com Kenneth M. Price e Ray Siemens como editores, aos quais se juntaram, em 2014, Dene Grigar e Elizabeth Lorang como editoras associadas. Da primeira versão, disponibilizada em linha em 2013, constavam nove artigos de autoridades neste campo, aos quais, em 2015, se vieram acrescentar dois novos contributos. Atualmente, encontra-se aberta uma convocatória para novos artigos que se irão adicionar aos já publicados na plataforma. Os recursos disponibilizados em Literary Studies in the Digital Age são artigos dinâmicos e abertos a contributos da comunidade de autores e leitores. Tanto os artigos novos passam por um processo público de revisão por pares através da plataforma, como os artigos já aprovados estão abertos aos comentários e anotações dos leitores. A plataforma oferece, por isso, uma interessante ferramenta de leitura social distribuída, que procura novas estratégias e modelos colaborativos de produção de conhecimento.

Bibliografia relacionada:

Fitzpatrick, Kathleen (2011). Planned Obsolescence: Publishing, Technology, and the Future of the Academy. New York: NYU Press.

Gold, Matthew K., \& Lauren F. Klein, eds. (2016). Debates in the Digital Humanities 2016. Minneapolis, MN: Minnesota University Press. 
Mandell, Laura (2015). Breaking the Book: Print Humanities in the Digital Age. New Jersey: Wiley-Blackwell.

Meyer, Eric T., \& Ralph Schroeder (2015). Knowledge Machines: Digital Transformations of the Sciences and Humanities. Cambridge, MA: The MIT Press.

\section{Stanford Literary Lab \\ https:/ litlab.stanford.edu/ \\ Diretores: Franco Moretti e Mark Algee-Hewitt. Data de publica- ção: 2010-2016 [em curso]. Stanford University. Acesso aberto [portal com copy- right].}

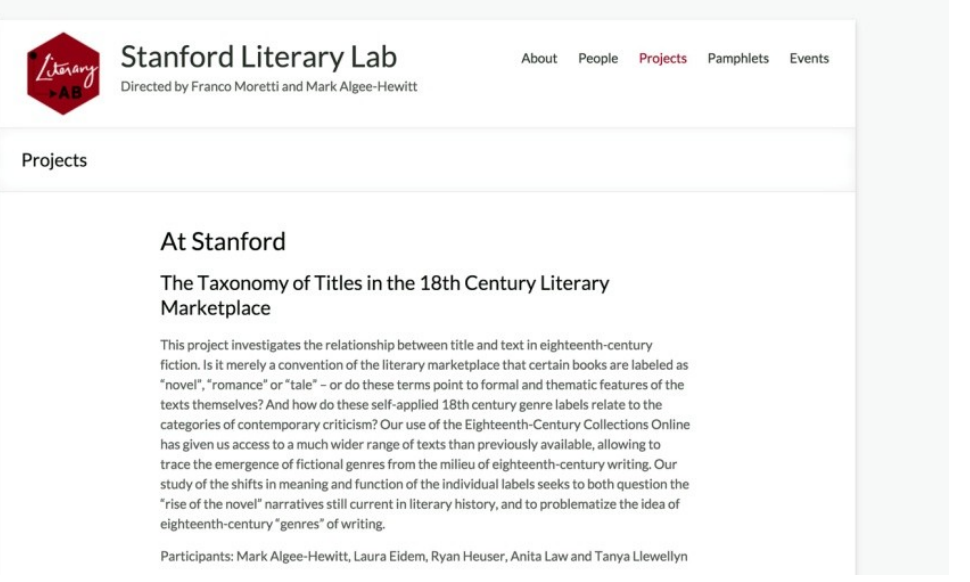

O Stanford Literary Lab é um laboratório constituído por investigadores que se dedicam à pesquisa colaborativa com base em técnicas quantitativas e macroanalíticas, de visualização de dados e de "leitura distante". Desde a sua fundação, em 2010, têm sido vários os projetos desenvolvidos pelo grupo, que conta com mais de quarenta investigadores da Universidade de Stanford. Alguns desses projetos têm sido realizados em parceria com outras universidades e instituições nacionais e estrangeiras. Os resultados desses projetos podem ser consultados e descarregados a partir do sítio web do Stanford Literary $L a b$, sendo que, em alguns casos, o utilizador pode ter acesso igualmente a alguns dos dados não tratados (raw data) recolhidos no âmbito dos projetos. Não só a produção final e os dados de trabalho dos projetos são disponibilizados em acesso livre, como o próprio laboratório se encontra aberto a investigadores que queiram colaborar nos projetos existentes ou propor novos projetos. Atualmente, encontram-se em curso no laboratório seis projetos sediados na Universidade de Stanford e quatro desenvolvidos em parceria com outras instituições. Os projetos interdisciplinares em curso abordam diversos temas, numa igualmente variada panóplia de estratégias, metodolo- 
gias e técnicas. A título de exemplo, The Taxonomy of Titles in the 18th Century Literary Marketplace é um projeto centrado na análise de um largo conjunto de dados extraídos de obras de ficção do século XVIII, tendo como objetivo entender a relação entre títulos, textos e géneros. Por seu turno, The Emotions of London tem como meta criar um mapa emocional da capital inglesa, cruzando lugares mencionados em romances dos séculos XVIII e XIX com a significância afetiva que aí lhes é atribuída.

Bibliografia relacionada:

Gitelman, Lisa, ed. (2013). "Raw Data" is an Oxymoron. Cambridge, MA: The MIT Press.

Jockers, Matthew Lee (2013). Macroanalysis: Digital Methods and Literary History. Urbana: University of Illinois Press.

Jockers, Matthew Lee, \& Ted Underwood (2016). "Text-mining the Humanities." A New Companion to Digital Humanities. Eds. Susan Schreibman, Ray Siemens \& John Unsworth. Chichester: WileyBlackwell. 291-306.

Moretti, Franco (2013). Distant Reading. London: Verso.

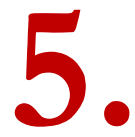

\section{TAPoR 3}

http://tapor-test.artsrn.ualberta.ca/home

Responsáveis: Geoffrey Rockwell, Stéfan Sinclair, Kirsten C. Uszkalo e Milena Radzikowska. Data de publicação: 2005 [versão 1], 2014 [versão 2], 2015 [versão 3]. McMaster University e University of Alberta. Acesso aberto [portal com copyright, softwares sob licenças diversas].

About Tour Contact Tools Lists Useful links Login

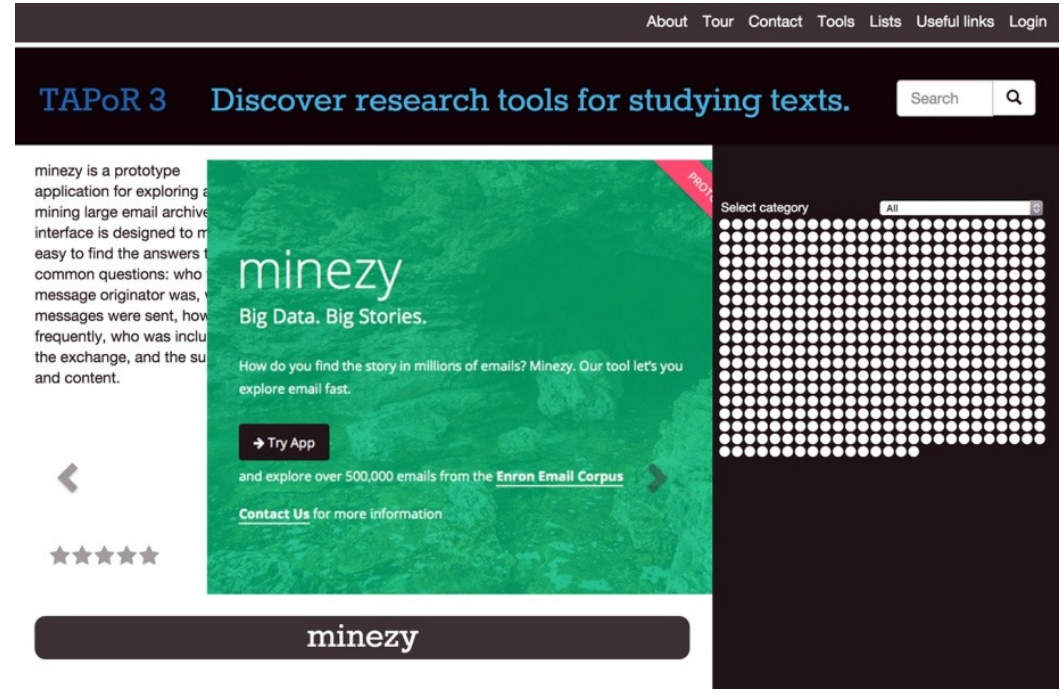

Atualmente na sua terceira versão, TAPoR (Text Analysis Portal for Research) é um diretório que faculta uma síntese descritiva de software de 
análise, manipulação e visualização de texto. Abrange ferramentas atuais e históricas (desenvolvidas antes de 2005), sendo cada entrada acompanhada por um sistema organizado de atributos e etiquetas descritivas. É possível pesquisar as ferramentas de acordo com critérios como tipo de análise textual, se se trata de uma aplicação web ou de instalação local, frequência de utilização, facilidade de utilização, tipo de licença, grupo ou laboratório que a produz e mantém, etc. Todos os conteúdos do diretório estão abertos à edição colaborativa mediante criação de conta de utilizador, sendo ainda possível ler comentários e recomendações de outros utilizadores, bem como seguir publicações externas sobre as ferramentas descritas. Trata-se de um recurso essencial para conhecer as tecnologias atuais de análise computacional de texto.

Bibliografia relacionada:

Jockers, Matthew L. (2014). Text Analysis with R for Students of Literature. New York: Springer.

Kuckartz, Udo, \& Anne McWhertor (2014). Qualitative Text Analysis: A Guide to Methods, Practice \& Using Software. Los Angeles: SAGE.

Paulus, Trena M., Jessica Nina Lester, \& Paul G. Dempster (2014). Digital Tools for Qualitative Research. Los Angeles: SAGE.

\section{Text Encoding Initiative}

http://www.tei-c.org/index.xml

Diretores atuais: Michelle Dalmau, Marjorie Burghart, Martin Mueller, Kathryn Tomasek, Pip Willcox. Data de publicação: 1987-2016 [em curso]. Consórcio composto por várias instituições. Parceiros institucionais atuais: University of Oxford e TGIR Huma-Num. Acesso aberto [softwares de código aberto, distribuidos sob licenças Creative Commons e BSD-2].

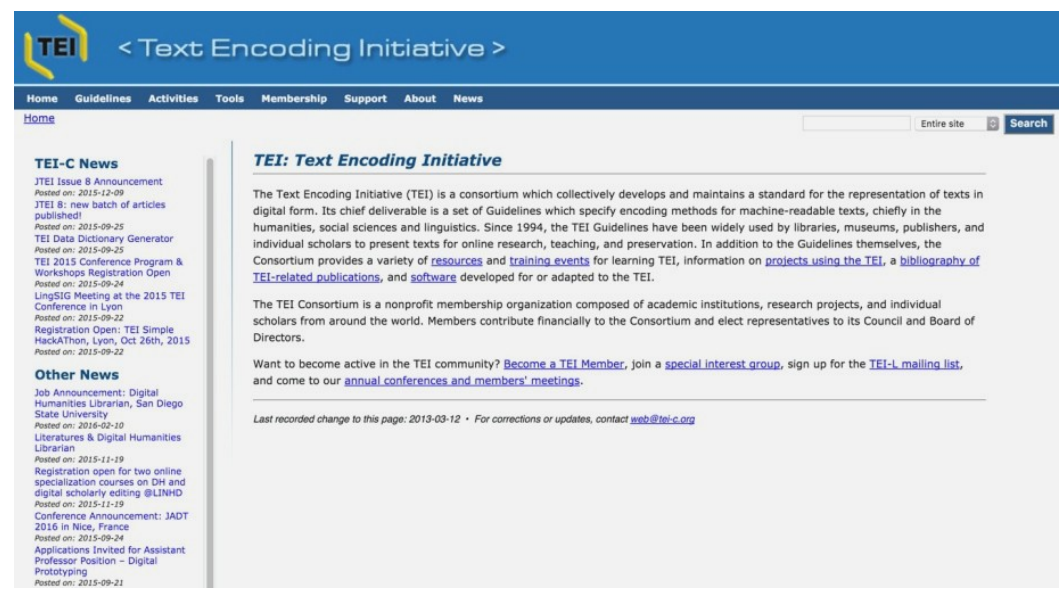


Desenvolvida desde o final dos anos 1980 por um consórcio que reúne bibliotecas, universidades, centros de investigação, entre outras entidades, a Text Encoding Initiative (TEI) apresenta uma norma internacional para a representação de textos sob forma digital. A primeira versão completa do manual (TEI P3) foi publicada em 1994 e, desde então, têm sido várias as reformulações às especificações e normas convencionadas, incluindo a adição de novos módulos. A norma atual (TEI P5), publicada em maio de 2011, encontra-se na sua versão 2.9 (outubro de 2015). A TEI tem, na sua base, o objetivo de fornecer um standard claro e simples, que permita aos investigadores apresentar de forma rigorosa os textos que são objeto dos seus projetos. A marcação e codificação de texto segundo as normas TEI exclui a vinculação a softwares específicos e garante a acessibilidade, longevidade e integridade intelectual dos textos representados. O esforço de criação de uma norma para a codificação textual vai, por isso, no sentido de garantir a interoperabilidade e a preservação futura do conhecimento em formatos legíveis por máquinas. Para além das normas e manuais a que é possível aceder no portal TEI, o sítio web fornece ainda um conjunto organizado de informação sobre ferramentas, publicações, atividades, workshops e projetos que fazem uso da codificação TEI.

Bibliografia relacionada:

Burnard, Lou (2014). What is the Text Encoding Initiative? How to Add Intelligent Markup to Digital Resources. Marseille: OpenEdition Press.

Walsh, John, ed. (2011-2016). Journal of the Text Encoding Initiative. Issues 1-8, http://jtei.revues.org/

Pierazzo, Elena (2016). "Textual Scholarship and Text Encoding." $A$ New Companion to Digital Humanities. Eds. Susan Schreibman, Ray Siemens \& John Unsworth. Chichester: Wiley-Blackwell. 307-321. 


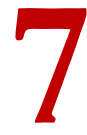

\section{Annotation Studio}

http://www.annotationstudio.org/

Diretores: Jim Paradis e Kurt Fendt. Data de publicação: 2014-2016 [em curso]. MIT School of Humanities, Arts and Social Sciences. Acesso aberto [aplicação web de código aberto, distribuído sob licença GPL2].

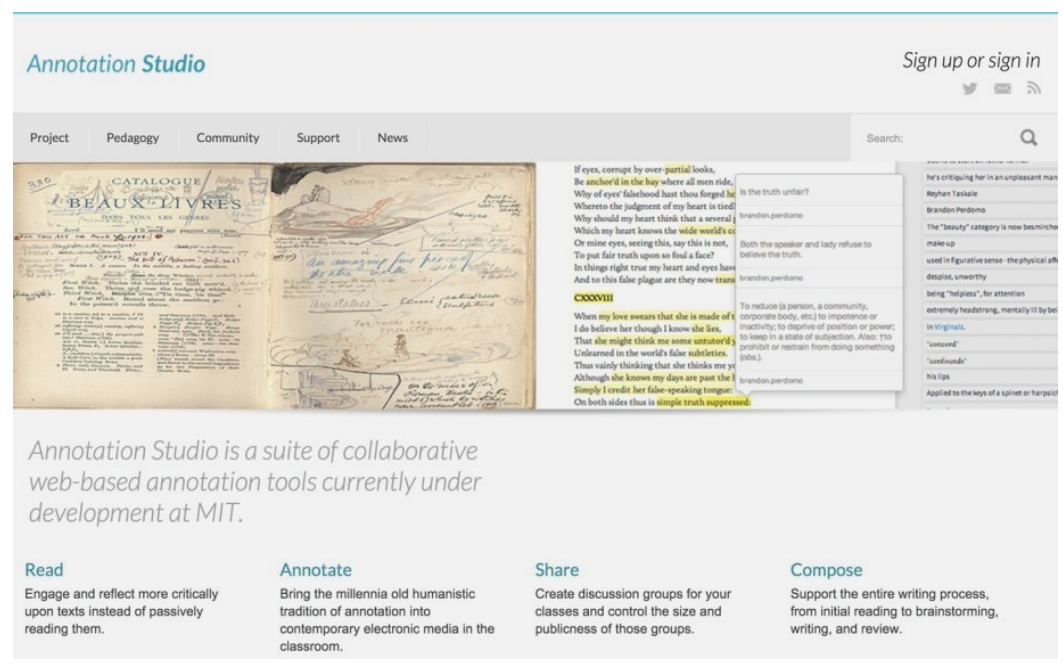

Annotation Studio é uma aplicação web desenvolvida pelo HyperStudio e tem como finalidade a anotação e comentário colaborativo de textos. Criada como ferramenta pedagógica de fácil utilização para estudantes, a aplicação não exige conhecimento de TEI ou outras normas específicas de marcação e codificação. A ferramenta oferece diversas técnicas relevantes para a interação em contexto de aprendizagem, servindo comunidades de estudantes e professores que se dedicam à leitura e comentário de um mesmo texto. Annotation Studio providencia aos professores ferramentas para o registo do processo de leitura dos seus alunos. Através de visualizações gráficas, é possível, por exemplo, destacar quais as passagens do texto com mais interação, ou criar mapas com percursos de leitura entre diferentes partes do texto. Embora os responsáveis pelo desenvolvimento da aplicação se tenham focado especialmente no contexto pedagógico, investigadores, bibliotecários e arquivistas são alguns outros grupos destacados como público no seu portal web.

Bibliografia relacionada:

Constance Crompton, Daniel Powell, Alyssa Arbuckle, Ray Siemens e Maggie Shirley (2014). "Building a Social Edition of the Devonshire Manuscript." Renaissance and Reformation, 37. 4: 131-156.

Price, Kenneth M. (2016). "Social Scholarly Editing." A New Companion to Digital Humanities. Eds. Susan Schreibman, Ray Siemens \& John Unsworth. Chichester: Wiley-Blackwell. 137-149. 


\section{8.}

Media Archaelogy Lab

http:/ /www.mediaarchaeologylab.com/

Diretora: Lori Emerson. Data de publicação: 2009-2016 [em curso].

University of Colorado at Boulder. Acesso aberto [portal com copyright].

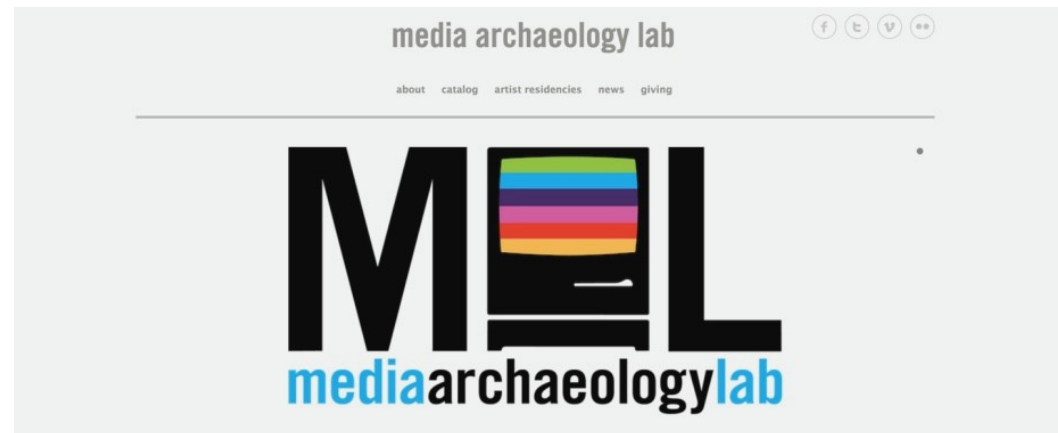

O Media Archaelogy Lab é um laboratório dedicado à investigação e ensino e é composto por um largo acervo de hardware e software obsoletos. O espaço conta com computadores, de secretária e portáteis, de várias épocas e fabricantes variados. Fazem também parte da coleção máquinas de escrever, processadores de texto, consolas de jogos, entre inúmeros periféricos. $\mathrm{O}$ acervo de software abrange pacotes de produtividade, de programação e recursos pedagógicos, mas também jogos, obras de ficção interativa e utilitários criados por utilizadores. O objetivo principal do Media Archaeology Lab é garantir a preservação e acesso a momentos cruciais na história da computação e da literatura digital. Procura, assim, por meio da arqueologia dos meios garantir que, preservando a memória histórica dos média, são criadas as condições para compreender a cultura digital numa perspetiva técnica e estética de longa duração.

Bibliografia relacionada:

Chun, Wendy Hui Kyong (2011). Programmed Visions: Software and Memory. Cambridge, MA: MIT Press.

Parikka, Jussi (2012). What is Media Archaelogy? London: Polity.

Parikka, Jussi (2015). A Geology of Media. Minneapolis: University of Minnesota Press.

Price, Katie L. (2015). "Quick question: How can media archeology inform literary studies?” Jacket 2. https://jacket2.org/commentary/howcan-media-archeology-inform-literary-studies 